\title{
Algorithm of Five-Axis Machining Tool Axis Vector Interpolation Based on NURBS Surface
}

\author{
Cheng De-rong, Deng Shi-ping and Xing Xiao-lin \\ (School of Mechatronic Engineering, Chongqing Technology and Business \\ Institute, Chongqing, China, 400052) \\ chengderong@cqdd.cq.cn,1615981897@qq.com,xingxiaol57@126.com
}

\begin{abstract}
To address the non-linear error and tool collision or tool interference introduced by the rotation axis, control strategy of five-axis machining tool axis vector interpolation based on NURBS surface is proposed. By using NURBS surface reconstruction ideas, after node vectors are inverse computed, it is inserted in the $U$ and V-parameter direction of the surface. Then surface control points are anti-calculated, and the data points of shape values on the surface are determined which is tool cutting contacts point. By using normal vector of surface and combining machine tool kinematics analysis results, the rotation angle of the two rotary axes are calculated. And then, the tool axis vector to be interpolated is determined. Simulation results show that the algorithm can effectively improve the accuracy of five-axis CNC machining, and verify the effectiveness and practicality of the algorithm.
\end{abstract}

Keywords: Five-axis machining, Tool axis vector, Insert node, Surface normal vector

\section{Introduction}

Five-axis $\mathrm{CNC}$ technology is an integration of precision machining and computer control and high-performance servo drive technology. Five-axis CNC machine tools introduces two rotary axes on the basis of the three-axis CNC machine tools. Therefore, five-axis CNC machine tools complete the machining process with more flexibility, and make the tool geometry and the workpiece surface achieve a better match. Five-axis machining is an important means to solve the complex surface machining. However, the introduction of two rotary axes increases the complexity of the five-axis CNC machine tool structure and the difficulty to control the posture of the tools. In actual machining process, CNC system drive rotary axis interpolation motion according to CAM system generating tool axis vector. There are two main ways to interpolation which are linear interpolation and tool axis vector interpolation. Linear interpolation is very easy to conduct while it results in more nonlinear errors. Tool axis vector interpolation is the result of interpolation operation based on machining needs by using CAM system to generate tool axis vector. It can be determined the movement of each axis under the machine coordinate system after passing through post-processing machine.

Many scholars have conducted a number of studies on tool axis vector interpolation of five-axis machining. Geng Cong et al. [1] proposed a cutter path control algorithm by controlling the movement of the first tool axis vector and the end of tool axis vector on the plane defined, in order to reduce the non-linear error. Fan Liuqun et al. [2] studied linear interpolation principle of the angle of rotation axis and what causes nonlinear machining errors, and proposed specific algorithm of tool axis vector interpolation based on tool axis vector plane, thus avoided the non-linear errors. However his method cannot guarantee the continuity of the speed and acceleration of rotating axis. Ji Junfeng, et al. [3] studied tool axis vector generation and smooth method using five-axis NC machining for the open impeller overall. Jun et al. [4] proposed a C-space tool axis vector algorithm 
and optimization, which can effectively prevent the angle from excessively changing between the adjacent tool axis. Chen Liangii et al. [5] proposed a linear interpolation of tool axis vector on both sides of the impeller pressure side and suction surface finishing. Cai Yonglin [6] proposed to use five times NURBS curve fitting the upper and lower boundaries of the original surface machining toolpaths edge, and sequentially connecting the upper and lower data points with shape in the curve. It can get $\mathrm{C} 2$ continuous cutter curve and the smoothly-changing spatial tool axis vector.

Machine vibration can be caused by discontinuous velocity and acceleration of the rotation axis, plus acceleration step change and so on. This will affect the surface quality and surface roughness. Therefore, the key issue that five-axis NC machining tool position control need to address is how to complete rotary axis tool axis vector interpolation. In this regard, we propose a control strategy about interpolation tool axis vector based on NURBS surfaces.

\section{Nurbs Surface Reconstruction Interpolation Algorithm Idea}

The traditional interpolation method is to calculate intermediate point coordinates between the starting and ending point of the contour lines, which is in nature to the encrypt data points. Data sampling interpolation method is widely used in the modern CNC system. In the interpolation process, if the curve is approximated by a straight line segment, CNC system will not only increase the amount of storage, but also will destroy the continuity of the original curve. Then it will cause that interpolation speed and acceleration will become discontinuous and its plus acceleration will happen step change. Thus, it will lead to the occurrence of a large machine vibration and shock, and it will affect on parts of the surface quality and reduce machining speed. In this regard, we propose a tool axis vector interpolation method using surface interpolation, by using NURBS surface reconstruction ideas, after node vectors are inverse computed, it is inserted in the $\mathrm{U}$ and $\mathrm{V}$-parameter direction of the surface. Then surface control points are anti-calculated, and the data points of shape values on the surface are determined which is tool cutting contacts point. By using normal vector of surface and combining machine tool kinematics analysis results, the rotation angle of the two rotary axes are calculated. And then, the tool axis vector to be interpolated is determined. In the process of running the tool axis vector, the tool axis vector will be always kept on sector surface boundary with start and end tool axis vector within the program block, and be maintained the tool axis vector velocity and acceleration continuity. Ultimate we can achieve to control tool smooth machining and reducing non-linear errors.

\section{Interpolation Algorithm Based On NURBS Surfaces}

\subsection{NURBS Surfaces in the Form of Expression}

Similar to NURBS curves, NURBS surfaces can be represented in precise mathematical expression.

$$
C(u, v)=\frac{\sum_{i=0}^{m} \sum_{j=0}^{n} w_{i, j} q_{i, j} N_{i, k}(u) N_{j, l}(v)}{\sum_{i=0}^{m} \sum_{j=0}^{n} w_{i, j} N_{i, k}(u) N_{j, l}(v)}
$$

In the formula (1), ${ }^{q_{i, j}}$ is the control point on NURBS surfaces. It is a rectangular array of topological spatial distribution. $w_{i, j}$ is the weighting factor associated with $q_{i, j}$. Weighting factors in the right vertex $w_{0,0}, w_{0, n}, \quad w_{m, 0}$ and ${ }^{w_{m, n}}$ are greater than 0 . The 
rest weighting factor $w_{i, j}$ is greater than and equal to $0 . N_{i, k}(u)$ is a k times of B-spline basis function in the $\mathrm{U}$-direction. It is a $\mathrm{k}$ times of piecewise polynomial determined by $U=\left[u_{0}, u_{1}, \ldots \ldots, u_{m+k+1}\right]$ node vector. $N_{j, l}(v)$ is a 1 times of B-spline basis function in the V-direction. It is a 1 times of piecewise polynomial determined by $V=\left[v_{0}, v_{1}, \ldots \ldots, v_{n+l+1}\right]$ node vector. These two nodes vectors can work out by applying the Boer-Cox recurrence formula.

$$
\left\{\begin{array}{l}
N_{i, 0}(u)= \begin{cases}1 & u_{i} \leq u \leq u_{i+1} \\
0 & \text { Other }\end{cases} \\
N_{i, k}(u)=\frac{u-u_{i}}{u_{i+k}-u_{i}} N_{i, k-1}(u)+\frac{u_{i+k+1}-u}{u_{i+k+1}-u_{i+1}} N_{i+1, k-1}(u) \\
\text { Provision }: \frac{0}{0}=0
\end{array}\right.
$$

\subsection{OK to Insert the Node Vector}

Formula (2) shows that NURBS surfaces shape is associated with the control point, the weighting factor and the node vectors. Therefore, to modify the shape of NURBS surfaces, we need to insert a node vector and modify weighting factor and modify the control point coordinate.Formula (1) can be written as follows:

$$
C(u, v)=\frac{\sum_{i=0}^{m} N_{i, k}(u) \sum_{j=0}^{n} w_{i, j} q_{i, j} N_{j, l}(v)}{\sum_{i=0}^{m} N_{i, k}(u) \sum_{j=0}^{n} w_{i, j} N_{j, l}(v)}
$$

When v certain value $v_{j}(j=0,1,2, \ldots \ldots, n)$, formula (3) can be expressed as formula (4).

$$
\left.C(u, v)\right|_{v=v_{j}}=\frac{\sum_{i=0}^{m} N_{i, k}(u) W_{j}^{v} q_{i, j}}{\sum_{i=0}^{m} N_{i, k}(u) W_{j}^{v}}
$$

In the formula, $W_{j}^{v}=\sum_{j=0}^{n} w_{i, j} N_{j, l}(v)$

When u certain value $u_{i}(i=0,1,2, \ldots \ldots, m)$, formula (3) can be expressed as formula (5).

$$
\left.C(u, v)\right|_{u=u_{i}}=\frac{\sum_{j=0}^{n} N_{j, l}(v) S_{i}^{u} q_{i, j}}{\sum_{j=0}^{n} N_{j, l}(v) S_{i}^{u}}
$$

In the formula,

$$
W_{j}^{v}=\sum_{i=0}^{m} w_{i, j} N_{i, k}(u) .
$$

It can be seen formula (4) and formula (5) are essentially represented as a NURBS curve. That is, if a series of values of any parameter $v$ is determined, NURBS curve group will be formed in U-direction about the parameter u. As shown in Figure 1. 


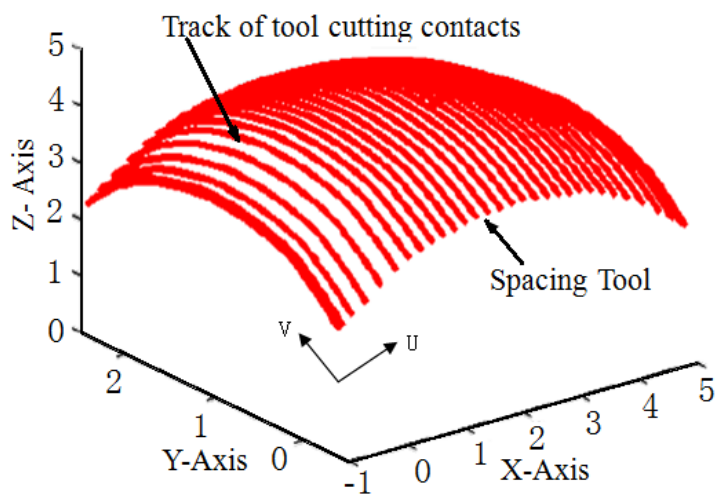

Figure 1. Discrete NURBS Surfaces by Equal Parameters Methods

In order to accomplish anti-plug node vector on NURBS surfaces, inserting nodes vector on NURBS surfaces is similar to NURBS curve insert node vector technology. That is, after the control point are inserted the control grid, computing node vector is to be inserted in turn.

Suppose the new node vector $q_{u}$ is inserted on the surface of the control grid of $q_{i, j} q_{i+1, j}$ edge in U-direction. Vertices of the control grid is four point of $q_{i, j}, q_{i, j+1}, q_{i+1, j}$ and $q_{i+1, j+1}$. The results are as follows.

$$
q_{u}=\frac{(1-\lambda) w_{i, j} q_{i, j}+\lambda w_{i+1, j} q_{i+1, j}}{(1-\lambda) w_{i, j}+\lambda w_{i+1, j}} \quad \lambda=\frac{w_{i, j} \overline{q_{u} q_{i, j}}}{w_{i, j} \overline{q_{u} q_{i, j}}+w_{i+1, j} \overline{q_{u} q_{i+1, j}}}
$$

According to insert node vector technology in the NURBS curve, we can get the inserted node vector $u$ on NURBS surfaces. That is formula (6).

$$
u=u_{i+1}+\lambda\left(u_{i+k+1}-u_{i+1}\right)
$$

Similarly, the new node vector $q_{v}$ is inserted in the $\mathrm{V}$ direction of the surface of the control grid of $\overline{q_{i, j} q_{i, j+1}}$ edge. Node vector v can be obtained as in formula (7).

In the formula, $\quad w_{i, j} \overline{q_{v} q_{i, j}}+w_{i, j+1} \overline{q_{v} q_{i, j+1}}$.

\subsection{Generate aControl Point}

Then insert a new node vectors $\mathrm{u}$ in the $\mathrm{U}$-direction and insert a new node vectors $\mathrm{v}$ in the $\mathrm{V}$-direction. The results are to add a control point in each row in the U-direction and $\mathrm{V}$-directions. The intersecting point of the U-direction of NURBS curves and of the Vdirection of NURBS curves is control Point $q_{i j}$. It can be solved for the coordinate value of the control point $q_{i j}$ after putting the formula (6) and formula (7) into the formula (4) and formula (5). As shown in figure 2, the number of control point of NURBS surfaces turns nine from the original four. As shown in figure 3, the number of control point of NURBS surfaces turns twenty five from the original nine. 


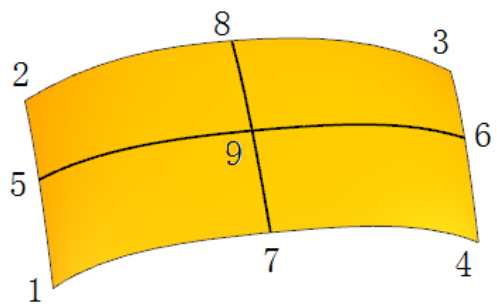

Figure 2. Surface Interpolation Scheme 1

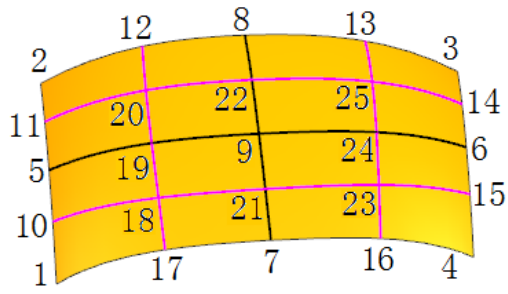

Figure 3. Surface Interpolation Scheme 2

After putting the node vectors values of the formula (6) and formula (7) into the formula (2), it can determine that the basis function $N_{i, k}(u)$ and $N_{j, l}(v)$. To simplify the calculation, all weighting factor defaults to 1 in this algorithm. After putting the coordinate values of control points $q_{i j}$ and the basis function $N_{i, k}(u)$ and the basis function $N_{j, l}(v)$ into the formula (1), the data points with shape can be obtained on the reconstructed surface. This data points with type value is machining of tool cutting contacts.

\subsection{NURBS Surface Normals Vector}

For NURBS surfaces $C(u, v)$, if the fixed parameters u equals to $u_{0}$, the surface $C(u, v)$ becomes a unary function $C\left(u_{0}, v\right)$ about parameters v. It indicates a NURBS curves about parameter v on a NURBS surface, which is called v-line. Similarly, if the fixed parameters $\mathrm{v}$ equals to $v_{0}$, the surface $C(u, v)$ becomes a unary function $C\left(u, v_{0}\right)$ about parameters $u$. It indicates a NURBS curves about parameter $u$ on a NURBS surface, is called u-line. Therefore, at any point $C\left(u_{0}, v_{0}\right)$ on the surface, there must be one u-line passing through this point and one v-line. At this point, the partial derivative vector $\left.C_{u}\left(u, v_{0}\right)\right|_{u=u_{0}}=\left.\frac{\partial C\left(u, v_{0}\right)}{\partial u}\right|_{u=u_{0}}$ of u-line about parameters $\mathrm{u}$ is called tangent vector in the $\mathrm{U}$-direction at the point. If the fixed parameters $\mathrm{v}$ equals to $v_{0}$, the partial derivative vector $C_{u}\left(u, v_{0}\right)=\frac{\partial C\left(u, v_{0}\right)}{\partial u}$ of u-line about parameters $\mathrm{u}$ is called tangent vector in the Udirection of $\mathrm{u}$-line. Therefore, if the fixed parameters $\mathrm{u}$ equals $u_{0}$, the partial derivative vector $C_{v}\left(u_{0}, v\right)=\frac{\partial C\left(u_{0}, v\right)}{\partial v}$ of v-line about parameters $\mathrm{v}$ is called tangent vector in the Vdirection of v-line. If tangent vector in the $\mathrm{U}$-direction and tangent vector in the $\mathrm{V}$ - 
direction are not parallel, that is $C_{u}\left(u_{0}, v_{0}\right) \times C_{v}\left(u_{0}, v_{0}\right) \neq 0$, it can deduce the unit normal vector $n\left(u_{0}, v_{0}\right)$ from the tangent plane at the point $C\left(u_{0}, v_{0}\right)$ on NURBS surfaces. It can be illustrated in the following formula:

$$
n\left(u_{0}, v_{0}\right)=\frac{C_{u}\left(u_{0}, v_{0}\right) \times C_{v}\left(u_{0}, v_{0}\right)}{\left|C_{u}\left(u_{0}, v_{0}\right) \times C_{v}\left(u_{0}, v_{0}\right)\right|}
$$

Therefore, the unit normal vector of tool cutting contacts on the surface $C(u, v)$ is as follows:

$$
\begin{aligned}
& n_{i j}\left(u_{i}, v_{j}\right)=\frac{C_{u}\left(u_{i}, v_{j}\right) \times C_{v}\left(u_{i}, v_{j}\right)}{\left|C_{u}\left(u_{i}, v_{j}\right) \times C_{v}\left(u_{i}, v_{j}\right)\right|} \\
& n_{i j}\left(u_{i}, v_{j}\right) \text { can also be expressed as } n_{i j}\left(n_{x}, n_{y}, n_{z}\right) .
\end{aligned}
$$

\subsection{Determining NURBS Surface Interpolation Step}

Based on the content of Chapter 2.2, if a series of the values of any parameter $\mathrm{v}$ is determined, surfaces $C(u, v)$ will be discreted into NURBS curve groups about parameter $\mathrm{u}$ in the U-direction, as shown in figure 1 . If a series of values of the parameters $\mathrm{v}$ is to be determined, and equal spacing is taken spacing between adjacent curves, we call it the method of equal parameters line discrete surface. When we use equal parameters line method discrete locus of NURBS surfaces $C(u, v)$, the smaller the value parameters u and $\mathrm{v}$ is, the higher the precision. But, if the value of parameter $\mathrm{u}$ and $\mathrm{v}$ is too small, it would greatly increases the computation time and data storage, and reduces processing speed. Therefore, it is essential to determine the appropriate step size in the U-direction and Vdirections.

According to Cutting principle, if it is assumed that cutting direction of cutting line is the direction u-parameter, feed direction of the cutting line is the direction v-parameter. As shown in figure 1 . When parameter v equal to $v_{j}(\mathrm{j}=0,1, \cdots, \mathrm{n})$, NURBS surfaces can be abbreviated as $C(u)$.It is represented as a series of equal parameters line as the u-line. When parameter v equal to $v_{j}(\mathrm{j}=0)$, the u-line is a curve. If it take two points of $u_{0}$ and $u_{1}$ in this curve, 5 -axis machining for surface will produce chord error $\varepsilon$ due to linear approximation. As shown in figure 4, known as chord error $\varepsilon=\frac{L_{u}{ }^{2}}{8} \frac{1}{\rho}$, among them $L_{u}=C\left(u_{1}\right)-C\left(u_{0}\right)$, character $\rho$ is the radius of curvature when parameter u equal to $u_{0}$ in the curve $C(u)$. Processing step in the U-direction is represented by $L_{u}$ and $L_{u}=\sqrt{8 \rho \varepsilon}$.

Tool feed spacing refers to spacing between the line that after the finish line cutting tool to the line that steering cutting next line. Spacing value is related to precision. To simplify the calculations, when the tool axis real-time interpolation, replace surface with plane in part of the area. For example, in the course of CNC milling using the ball milling, residual height is represented by character $\mathrm{h}$ and $h=R-\sqrt{R^{2}-\frac{L_{v}{ }^{2}}{4}}$ tool radius. As shown in Figure 5. Determine the limits of residual height $h_{\max }$ according to the machining precision. Thus calculate the processing step $L_{v}$ and $L_{v}=2 \sqrt{2 R h_{\max }-h_{\max }^{2}}$ 


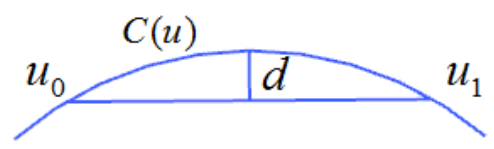

Figure 4. Diagram of Chord Error

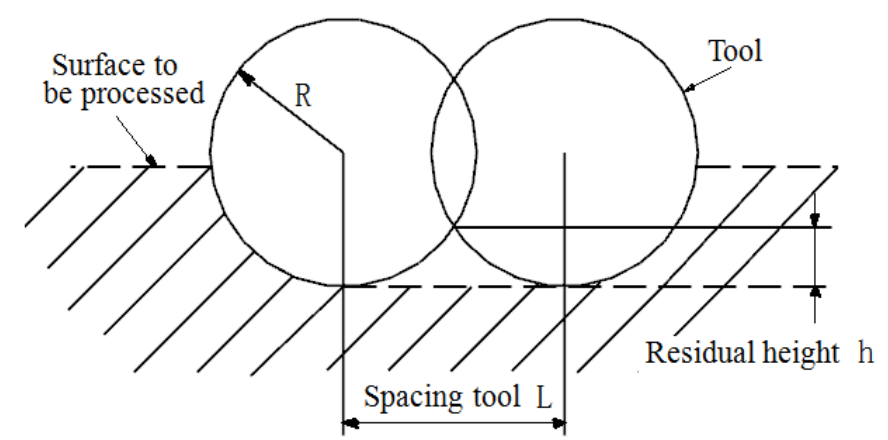

Figure 5. Spacing Tool And Residual Height

\section{Solving Two Rotary Axes}

To achieve five-axis machining, it is is necessary to calculate the position of the two rotary axes or rotation angle. In addition, we also need to obtain the position of interpolation point. Here in $\mathrm{A}, \mathrm{C}$ double turntable rotation axis is an example. With the change of the curved shape, it is difficult to achieve the goal of controlling the cutter shaft along adaptively adjusting. In the five-axis surface machining process, there are three states between the tool axis and the surface: vertical, parallel, and at an angle. In either states, it is angled between the tool axis direction and the surface normal vector. Therefore, before controlling the tool axis direction, the normal processing of surfaces must be determined ${ }^{[7]}$. In reference to the NURBS surface theory, the unit normal vector of the complex surface can be obtained after the tangential vectors in the U-direction and in the V-direction are worked out.

In the tool coordinate system, assuming the A-axis rotational motion is represented by $\theta_{A}(u, v)$; and the $\mathrm{C}$-axis rotational motion represented by $\theta_{C}(u, v)$. Homogeneous coordinate transformation matrix of A-axis rotary motion represented by $R_{x}\left(\theta_{A}(u, v)\right)$. Homogeneous coordinate transformation matrix of C-axis rotary motion represented by $R_{z}\left(\theta_{C}(u, v)\right)$. If the unit surface normal vector of the initial value take $(0,0,-1), \mathrm{A}$-axis and $\mathrm{C}$-axis of the initial value is Zero. Then there are the following formulas.

$$
\begin{aligned}
& R_{x}\left(-\theta_{A}(u, v)\right)=\left[\begin{array}{cccc}
1 & 0 & 0 & 0 \\
0 & \cos \left(\theta_{A}(u, v)\right) & \sin \left(\theta_{A}(u, v)\right) & 0 \\
0 & -\sin \left(\theta_{A}(u, v)\right) & \cos \left(\theta_{A}(u, v)\right) & 0 \\
0 & 0 & 0 & 1
\end{array}\right] \\
& R_{z}\left(-\theta_{C}(u, v)\right)=\left[\begin{array}{cccc}
\cos \left(\theta_{C}(u, v)\right) & \sin \left(\theta_{C}(u, v)\right) & 0 & 0 \\
-\sin \left(\theta_{C}(u, v)\right) & \cos \left(\theta_{C}(u, v)\right) & 0 & 0 \\
0 & 0 & 1 & 0 \\
0 & 0 & 0 & 1
\end{array}\right]
\end{aligned}
$$




$$
n\left(n_{x}, n_{y}, n_{z}\right)=R_{x}\left(\theta_{A}(u, v)\right) R_{z}\left(\theta_{C}(u, v)\right)\left(\begin{array}{lll}
0 & 0 & -1
\end{array}\right)^{T}
$$

Thus the value of rotation angle of tool axis vector interpolation can be obtained based on NURBS surface. As shown in formula (9) and formula (10).

$$
\begin{gathered}
\theta_{A}(u, v)= \pm \arccos \left(n_{z}\right) \quad\left(-\pi \leq \theta_{A}(u, v) \leq \pi\right) \\
\theta_{C}(u, v)=\arctan \left(n_{x} / n_{y}\right) \quad \text { or } \theta_{C}(u, v)=\arctan \left(n_{x} / n_{y}\right)-\pi \quad\left(0 \leq \theta_{C}(u, v) \leq 2 \pi\right)
\end{gathered}
$$

Finally, according to the requirements of surface machining error, using the parameter domain and physical coordinates to-one mapping, using adaptive algorithm step in the directions of estimated parameters, it can generate the toolpath and improve processing quality and processing efficiency.

\section{Simulation Analysis and Conclusions}

As shown in Figure 6, the graphics are changes in figure that interpolation step change with interpolation process when the tool axis does interpolation motion along a curve of U-direction. The tool axis vector changes smoothly when it makes interpolation motion. There is no stepwise mutation in the process. Figure 7 is the chord error with interpolation process change map. Figure 8 is machining process error map. This machining method can controll the contour error of the curve within $0.016 \mathrm{~mm}$. From the simulation results, following the requirements of this algorithm we can obtain satisfactory results, and can effectively improve the accuracy of five-axis CNC machining. It also verified effectiveness of the algorithm and practicability. It verified that the tool axis vector is smooth, and that nonlinearity error is small.

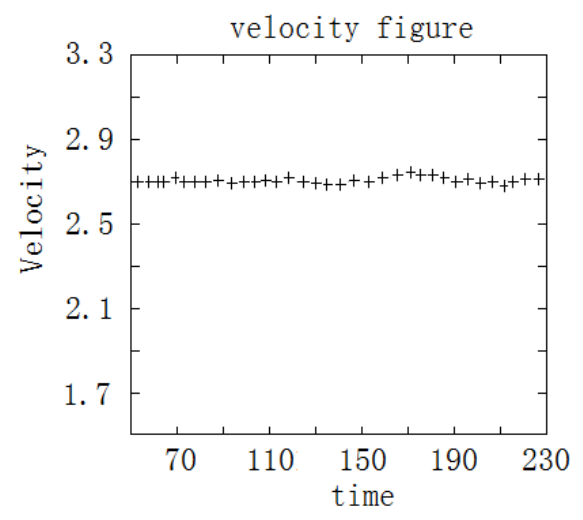

Figure 6. Step Change Map While Step 3

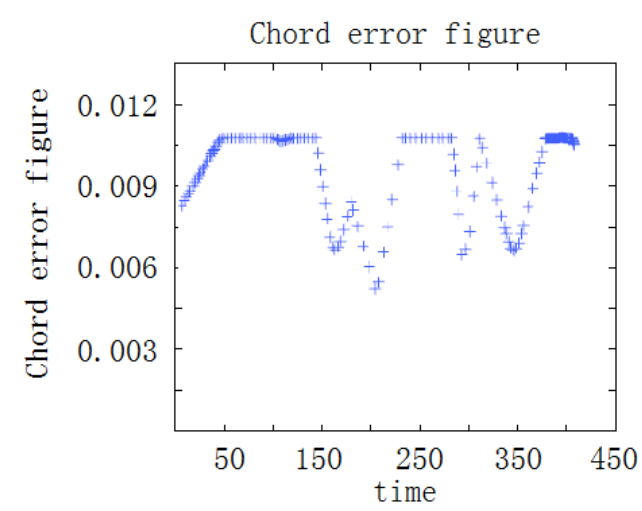

Figure 7. Chord Error Map 


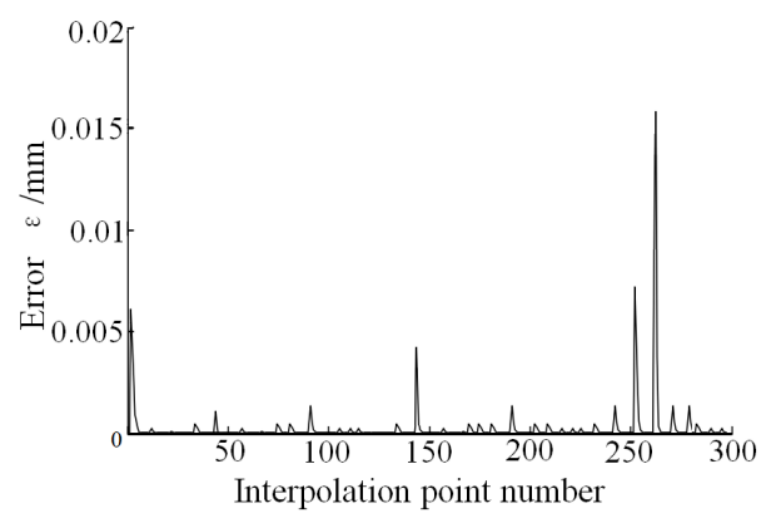

Figure 8. Machining Process Error Map

\title{
Acknowledgments
}

This research was supported by foundation of Chongqing municipal education commission of science and technology research project (No. KJ091063 and No. KJ1360612).

\section{References}

[1] G. Cong, D. Yu and X, Zhang, "Five-axis CNC machining tool path control algorithm", China Mechanical Engineering, vol. 21, no. 24, (2010), pp. 2904-2909.

[2] L. Fan, J. Qi, S. Bin and Z. Zhu, 'Plane Interpolation of Tool Orientation Algorithm for 5-axis Circumference Milling”, Mechanical Engineering, vol. 47, no. 19, (2011), pp. 158-162.

[3] J. Ji, "Research on Key Technology of NC Machining of Complex Integral Impeller", Nanjing University of Aeronautics and Astronautics, Nanjing, (2009).

[4] C.S. Jun, K. Cha, "Optimizing tool orientations for 5 - axis machining by configuration - space search method [J]", Computer, vol. 35, no. 6, (2003), pp. 549 - 566.

[5] L. J. Chen and X. Z. Feng, "Look-ahead controlling method of feed rate in 5-axis NURBS interpolation", Computer Integrated Manufacturing Systems, vol. 15, no. 12, (2009), pp. 2399-2403.

[6] C. Yonglin, S. Weiqing and J. Hong - "Detection for Interference in Numerical Control Machining of Impeller [J]", China Journal of Mechanical Engineering, vol. 18, no. 19, (2007), pp. 0- 2312.

[7] S. L. We, C. Wei and J. L. Gao, "Five-axis Interpolation Algorithm Base on NURBS Surface", Modular Machine Tool \& Automatic Manufacturing Technique, vol. 11, (2007), pp. 29-32.

\begin{abstract}
Authors
Cheng De-rong, she born in 1974 in Guang'an County, Sichuan Province, China. She is female. She is currently a master and an associate professor of School of Mechatronic Engineering of Chongqing Technology and Business Institute, China. The main research directions are the digital design and manufacturing.
\end{abstract}

Deng Shi-ping, he was born in 1963 in Chongqing, China. He is male. He is currently a Senior Engineer of School of Mechatronic Engineering of Chongqing Technology and Business Institute, China. He mainly engaged in the research and development of new functional materials and devices.

Xing Xiao-lin, he was born in 1957 in Huimin County, Shandong Province, China. $\mathrm{He}$ is male. He is currently a professor of School of Mechatronic Engineering of Chongqing Technology and Business Institute, China. The main research direction is the design and manufacture of mechanical, hydraulic and pneumatic technology. 
International Journal of Control and Automation

Vol.8, No.11 (2015)

Corresponding Author: Cheng De-rong

E-mail: chengderong@cqdd.cq.cn

Tel: +86 15909338946

Address: Room C107, Building Virtue, Si Yuan Road on the 15th, University Campus, Hechuan Area, Chongqing, China

Post Code: 401520 